\title{
Long Josephson junction embedded into a planar resonator at microwave frequencies: Numerical simulation of fluxon dynamics
}

\author{
E. Goldobin, ${ }^{\text {a) }}$ A. M. Klushin, M. Siegel, and N. Klein \\ Institut für Schicht- und Ionentechnik (ISI), Forschungszentrum Jülich GmbH (FZJ), \\ D-52425 Jülich, Germany
}

(Received 11 February 2002; accepted for publication 4 June 2002)

\begin{abstract}
We have investigated numerically a system consisting of a long Josephson junction embedded into a microstrip resonator. For such a configuration the Josephson vortex dynamics in the junction is driven by the oscillating currents in the resonator. We have calculated the complex impedance of the junction at the resonant frequency. The $Q$ factor of the resonator and the change of the resonant frequency of the whole system can then be easily calculated knowing the parameters of the resonator without Josephson junction. The fluxon dynamics and the complex impedance of the junction are simulated for different values of the junction length, damping, dc bias current, and dc magnetic field. This allows us to explain the behavior of the impedance of the junction and different loss mechanisms. In particular, we predict a nonmonotonic dependence of the both real and imaginary parts of the impedance on the amplitude of the oscillating current in the resonator for some range of parameters. Our results show that such a combination of active and passive superconducting elements forms a rather interesting nonlinear physical system which might be useful for development of tunable/switchable superconducting resonators and suggests an experimental technique for detecting trapped vortices in long junctions. (C) 2002 American Institute of Physics. [DOI: $10.1063 / 1.1497466]$
\end{abstract}

\section{INTRODUCTION}

The nonlinear losses in high- $T_{c}$ superconductors (HTS) at microwave frequencies or in the presence of the applied magnetic field can be explained by models in which HTS consists of superconducting grains connected by weak links acting like Josephson junctions. ${ }^{1}$ In order to verify this idea and to investigate it in detail, it was suggested ${ }^{2-6}$ to use HTS microstrip resonators with one large weak link formed by an artificial grain boundary (GB) extending over the entire width of the microstrip. Such a long Josephson junction (LJJ) is located in the middle of a linear resonator, so that the main mode of the resonator has the maximum amplitude of the oscillating current where the LJJ is placed. Theoretical as well as experimental investigations of such a system ${ }^{2-6}$ showed a very interesting fluxon (Josephson vortex) dynamics which until now was not fully investigated. In particular, the influence of the dc bias current, the dc magnetic field and the damping in LJJ on the fluxon dynamics, the $Q$ factor and the resonant frequency of the resonator was not studied yet. ${ }^{2-5}$ In Refs. 6 and 7 the dependence of these quantities on magnetic field was studied numerically and experimentally, but our results presented below are somewhat different and the complete understanding of experimental data presented in Refs. 6 and 7 has not yet been achieved.

Here we present the numerical study of the system in the same configuration as in Refs. 3 and 4, but extend our investigation to the more general case. Since the proposed system can also be fabricated using other types of Josephson junc-

${ }^{a)}$ Electronic mail: e.goldobin@fz-juelich.de, homepage: http://www.geocities.com/e_goldobin. tions (e.g., $\mathrm{Nb}-\mathrm{Al}-\mathrm{AlO}_{x}-\mathrm{Nb}$ in overlap geometry) with low damping (appreciable capacitance) and larger Josephson penetration length, we include a capacitive term into the model and study the influence of the damping on the behavior of the system.

Another reason for this investigation is to understand whether such a resonator can be tuned by an external magnetic field or by a dc bias current. Today, there is a huge interest in tunable or switchable superconducting resonators, so the investigation of this aspect of our system is definitely of interest.

This article is organized as follows. In Sec. II we present the model which we use for simulations and discuss the choice of parameters. The main results of the article and their discussion are presented in Sec. III. Section IV concludes the work.

\section{MODEL}

For simulation we use the STKJJ software. ${ }^{8}$ It implements an explicit finite difference algorithm for solving the perturbed sine-Gordon equation

$$
\phi_{x x}-\phi_{t t}-\sin \phi=\alpha \phi_{t}-P(x)\left[\gamma_{\mathrm{dc}}-\gamma_{\mathrm{ac}} \sin (\omega t)\right]
$$

which describes the dynamics of the Josephson phase $\phi(x, t)$ in a LJJ of length $L$ ( $x$ varies from 0 to $L$ ). Here and below the subscripts $x$ and $t$ denote partial derivative with respect to $x$ and $t$, accordingly. The spacial coordinate $x$ is normalized to the Josephson penetration length $\lambda_{J}=\sqrt{\Phi_{0} /\left(2 \pi \mu_{0} j_{c} d^{\prime}\right)}$ and the time $t$ is normalized to the inverse plasma frequency $\omega_{p}^{-1}=\sqrt{\Phi_{0} C /\left(2 \pi j_{c}\right)}$. In these definitions $j_{c}$ is the critical current density, $C$ is the specific capacitance of the Josephson 
barrier, and $d^{\prime}=d_{I}+2 \lambda \operatorname{coth}(d / \lambda) \approx 2 \lambda$ is proportional to the inductance of the superconducting electrodes of the LJJ, where $\lambda$ is the London penetration depth and $d$ is the thickness of the superconducting film forming the electrodes. The parameter $\alpha=1 / \sqrt{\beta_{c}}$ is the damping coefficient, $\beta_{c}$ $=\sqrt{2 \pi j_{c} R_{n}^{2} C / \Phi_{0}}$ is the McCumber-Stewart parameter, and $P(x)$ describes the spatial profile of the dc and ac normalized bias current densities $\gamma_{\mathrm{dc}}=j_{\mathrm{dc}} / j_{c}$ and $\gamma_{\mathrm{ac}}=j_{\mathrm{ac}} / j_{c}$. The spacial profile $P(x)$ of the dc and ac bias currents can be calculated numerically ${ }^{9}$ and supplied as input data. For the sake of simplicity, we use an approximate formula for thin films: ${ }^{10}$

$$
P(x) \propto \frac{1}{\sqrt{1-(2 x / L-1)^{2}}}
$$

with crossover to exponential dependence close to the edges. The crossover points $x=\lambda_{\text {eff }} / 2$ and $x=L-\lambda_{\text {eff }} / 2$ are defined by the parameter $\lambda_{\text {eff }}=\lambda^{2} / d$. For all our simulations we have used $\lambda_{\text {eff }}=0.05$ (in units of $\lambda_{J}$ ). $P(x)$ is normalized so that

$$
\int_{0}^{L} P(x) d x=1 .
$$

The boundary conditions for our linear, open ended geometry are

$$
\left|\phi_{x}\right|_{x=0}=\left.\phi_{x}\right|_{x=L}=h,
$$

where $h$ is the normalized magnetic field $h=2 H / H_{c 1}$, with $H_{c 1}=\Phi_{0} /\left(\pi \Lambda \lambda_{J}\right)$ being the Josephson vortex penetration field (field in the center of fluxon). The parameter $\Lambda=d_{I}$ $+2 \lambda \tanh (d / 2 \lambda) \approx 2 \lambda$ is the effective magnetic thickness of the junction.

The main difference between our model and the one used in Ref. 3 is that we do not skip the capacitive term $\phi_{t t}$ in Eq. (1), thus taking into account the small but finite capacitance of the junction. Therefore, we can define the Swihart velocity $\bar{c}_{0}=\lambda_{J} \omega_{p}$, which coincides with the maximum velocity of fluxon and equal to unity in normalized units. Using such model we can study the system in the overdamped $(\alpha \geqslant 1)$ as well as in the underdamped $(\alpha<1)$ limits.

Below we simulate the complex impedance $Z=R+i X$ of the LJJ at the resonator frequency as a function of the amplitude $\gamma_{\mathrm{ac}}$ of the ac current in the resonator. $R\left(\gamma_{\mathrm{ac}}\right)$ and $X\left(\gamma_{\mathrm{ac}}\right)$ are calculated $\mathrm{as}^{3}$

$$
\begin{aligned}
& R\left(\gamma_{\mathrm{ac}}\right)=\frac{2}{T \gamma_{\mathrm{ac}}} \int_{0}^{T} V_{\mathrm{eff}}(t) \sin (\omega t) d t, \\
& X\left(\gamma_{\mathrm{ac}}\right)=\frac{2}{T \gamma_{\mathrm{ac}}} \int_{0}^{T} V_{\mathrm{eff}}(t) \cos (\omega t) d t,
\end{aligned}
$$

where

$$
V_{\mathrm{eff}}(t)=\int_{0}^{L} V(x, t) P(x) d x
$$

represents an $x$-independent effective voltage across the LJJ. Knowing $R$ and $X$ of $\mathrm{LJJ}$, one can calculate the $Q$ factor and the changes of resonant frequency, if the parameters of the resonator without the embedded LJJ are known. Here we do not make these calculations because we want to keep our results as general as possible for further application to any particular type of the resonator.

In the normalized units introduced above, the voltage is expressed in terms of $\Phi_{0} \omega_{p} / 2 \pi$, i.e., in terms of the voltage corresponding to the plasma frequency, and the specific impedance will be expressed in the natural units of $\Phi_{0} \omega_{p} / 2 \pi j_{c}$. Note that such a definition of normalized specific impedance differs from the one used in Ref. 3. To convert our values to the values from Ref. 3 one has to multiply our values by $\alpha$.

All numerical results presented below are obtained for $\gamma_{\mathrm{dc}}=h=0, \alpha=1, L=20$ or 50 and $\omega=0.02$, if it is not specified otherwise. For typical values of plasma frequency $f_{p}$ $=\omega_{p} / 2 \pi=200 \mathrm{GHz}$ and $\lambda_{J}=2.5 \mu \mathrm{m}$, the corresponding physical values of $L$ will be 50 or $125 \mu \mathrm{m}, f=\omega / 2 \pi=4$ $\mathrm{GHz}, H_{c 1}=6.6 \mathrm{Oe}$, the unit of voltage will be $414 \mu \mathrm{V}$, and the unit of specific impedance will be $414 \Omega \mu \mathrm{m}^{2}$. The length is chosen so that the $\mathrm{JJ}$ is long $(L \gg 1)$ which corresponds to the experimental case, but in the same time not too long, in order to make the number of discrete elements $N$ $\sim L / \Delta x$ not too large and complete simulations during affordable time intervals. The choice of $\Delta x$ is discussed below. The resonator frequency is chosen to be 50 times as low as the plasma frequency of LJJ. On one hand, it should be low enough so that the fluxon can pass substantial distance along the LJJ during one period of oscillations in the resonator. If this condition is not satisfied, i.e., the driving frequency is of the order of the plasma frequency or higher, such a fast shaking of the fluxon may result in all kinds of odd behavior when the fluxon does not behave as a quasiparticle, e.g., emits plasma waves, etc. The sine-Gordon equation (1) and our simulation procedure describe the dynamics accurately in any case, but for clear interpretation of the results we stick with $\omega \ll \omega_{p}$. On the other hand, we cannot afford to make $\omega$ very small because we need to calculate the impedance by integrating the simulated average voltage over one period $T$ $=2 \pi / \omega$ [see Eqs. (4) and (5)], which can be rather time consuming for small $\omega$.

\section{RESULTS}

One of the important questions to consider is the choice of discretization $\Delta x$ which we use to solve Eq. (1) numerically. The authors of Ref. 3 claim that " $\Delta x=1$ (i.e., $\Delta x$ $=\lambda_{J}$ in physical units) results in a qualitatively correct solution." We argue that such a value of $\Delta x$ is too large. First, the adequate numerical approximation of the continuous solitonic solution of the sine-Gordon equation (1) is only possible if $\Delta x$ is much smaller than the soliton size, i.e., $\Delta x \ll 1$. In the underdamped case, we have to be especially careful since the soliton size can be quite small at high (relativistic) velocities $u \rightarrow 1$ due to the Lorentz contraction, i.e., the above condition becomes $\Delta x \ll 1 / \sqrt{1-u^{2}}$. If this condition is not satisfied, the numerical results correspond to the behavior of a parallel array of JJs described by a discrete sine-Gordon model. The dispersion relation in arrays is periodic and additional pinning of vortices in the cells is present. Second, the discretization of the sine-Gordon Eq. (1) in the vicinity of 

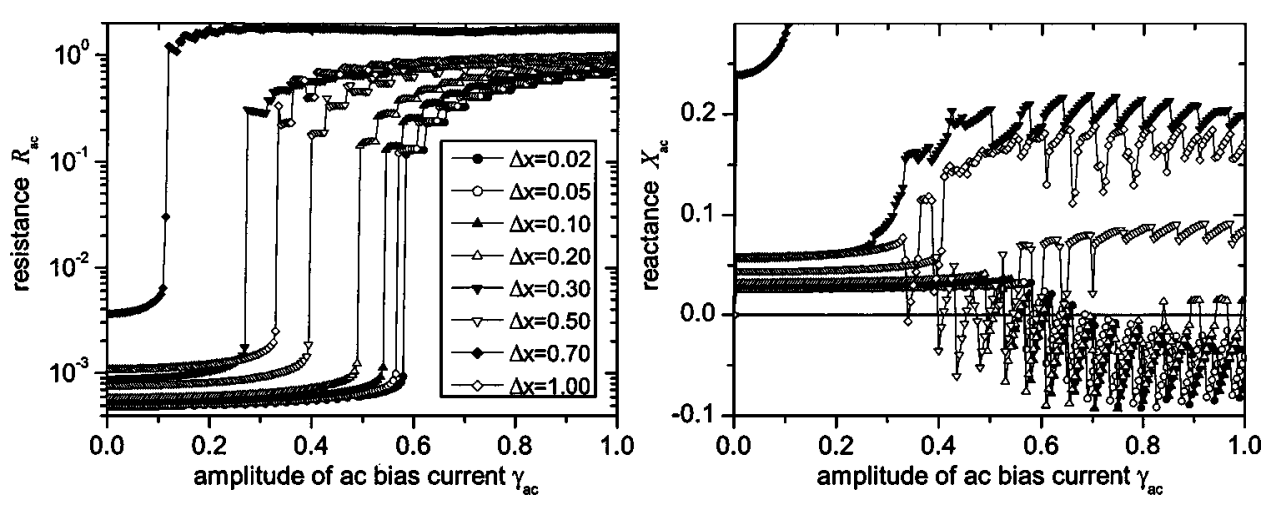

FIG. 1. The $R\left(\gamma_{\mathrm{ac}}\right)$ and $X\left(\gamma_{\mathrm{ac}}\right)$ dependencies for different discretization $\Delta x$ and for $L=20$ and $\omega=0.02$. the LJJ edges should be fine enough for adequate description of the fast spacial variation of $P(x)$. In particular, it is desirable that $\Delta x \ll \lambda_{\text {eff }}$, which for our choice of $\lambda_{\text {eff }}$ suggests very small values of $\Delta x \ll 0.05$. For a LJJ of the length $\sim 100$ this requires $\sim 10^{4}-10^{5}$ of discrete elements which should be processed during every time step of simulation. This results in unaffordable CPU time consumption.

To understand the impact of $\Delta x$ on the results of simulations we compare the calculated dependencies $R\left(\gamma_{\mathrm{ac}}\right)$ and $X\left(\gamma_{\mathrm{ac}}\right)$ obtained for various $\Delta x$ in the range from 0.02 to 1.0 as shown in Fig. 1. The physical interpretation of these curves is given below. Here it is only important that at small $\Delta x$ the curves converge to some limit which represents the behavior of a continuous system, while at $\Delta x>0.1$ they show very irregular and nonmonotonic (out-of-order) dependence on $\Delta x$. Therefore, for all further simulations we use $\Delta x=0.05$ which satisfies our first condition in all cases, except the case of very small damping $\alpha \leqslant 0.1$ when the velocity $u$ may be very close to 1 . The second condition $\Delta x$ $\ll \lambda_{\text {eff }}$ is not really satisfied (in our case $\Delta x=\lambda_{\text {eff }}$ ) but this results only in some shift of the $R\left(\gamma_{\mathrm{ac}}\right)$ and $X\left(\gamma_{\mathrm{ac}}\right)$ dependencies along the $\gamma_{\mathrm{ac}}$ axis towards lower values of $\gamma_{\mathrm{ac}}$, compare curves in Fig. 1 for $\Delta x=0.05$ and $\Delta x=0.02$. Such choice of parameters represents a good compromise between speed and accuracy.

\section{A. Finite capacitance}

The $R\left(\gamma_{\mathrm{ac}}\right)$ dependencies shown in Fig. 1 corresponding to small values of $\Delta x=0.02$ or 0.05 look very similar to that presented in Ref. 3. They have the same characteristic step structure related to the individual fluxon-antifluxon (F-AF) pair penetration into the LJJ, but the first $\mathrm{F}-\mathrm{AF}$ pair penetra- tion amplitude of ac bias current $\gamma_{\mathrm{ac}}^{p}$ is higher than in Ref. 3 due to the finer discretization $\Delta x$ in our case. On the other hand, the dependence $X\left(\gamma_{\mathrm{ac}}\right)$ looks rather different. In addition to the higher threshold mentioned above, $X\left(\gamma_{\mathrm{ac}}\right)$ does not grow approaching some finite positive value for large $\gamma_{\mathrm{ac}}$ as seen in Fig. 4 of Ref. 3, but rather has a saw-toothlike profile. Note that while the bias point moves along each step in the $R\left(\gamma_{\mathrm{ac}}\right)$ dependence, $X\left(\gamma_{\mathrm{ac}}\right)$ grows smoothly and almost linearly and can take positive (inductive behavior) as well as negative (capacitive behavior) values. This happens because we have included the capacitive term $\phi_{t t}$ into Eq. (1), while authors of Ref. 3 did not. Therefore, they obtained only positive (inductive) reactance while in our case $X\left(\gamma_{\mathrm{ac}}\right)$ can have any sign, and, in fact, it changes behavior from capacitive to inductive for the same number of trapped F-AF pairs. For GB LJJ used in Ref. 3 we calculated the value of $\alpha \approx 4$, which justifies the use of a model without a capacitive term.

\section{B. Length}

Now we study the dependence of $R\left(\gamma_{\mathrm{ac}}\right)$ and $X\left(\gamma_{\mathrm{ac}}\right)$ on the length of the LJJ. In Fig. 2 the family of $R\left(\gamma_{\mathrm{ac}}\right)$ and $X\left(\gamma_{\mathrm{ac}}\right)$ dependencies simulated for $L=2,5,10,20,50$, and 100 is shown. The longest junction has the lowest fluxon penetration amplitude $\gamma_{\mathrm{ac}}^{p} \approx 0.25$, since the current distribution in a wider strip has stronger current crowding peaks at the edges at $x=0$ and $x=L$. Thus the fluxons are injected easier into the longer LJJ. The steps on the $R\left(\gamma_{\mathrm{ac}}\right)$ dependence corresponding to different number of $\mathrm{F}-\mathrm{AF}$ pairs entering the junction tend to be larger (along $\gamma_{\mathrm{ac}}$ axis) in a longer system. The $X\left(\gamma_{\mathrm{ac}}\right)$ shows qualitatively different be-
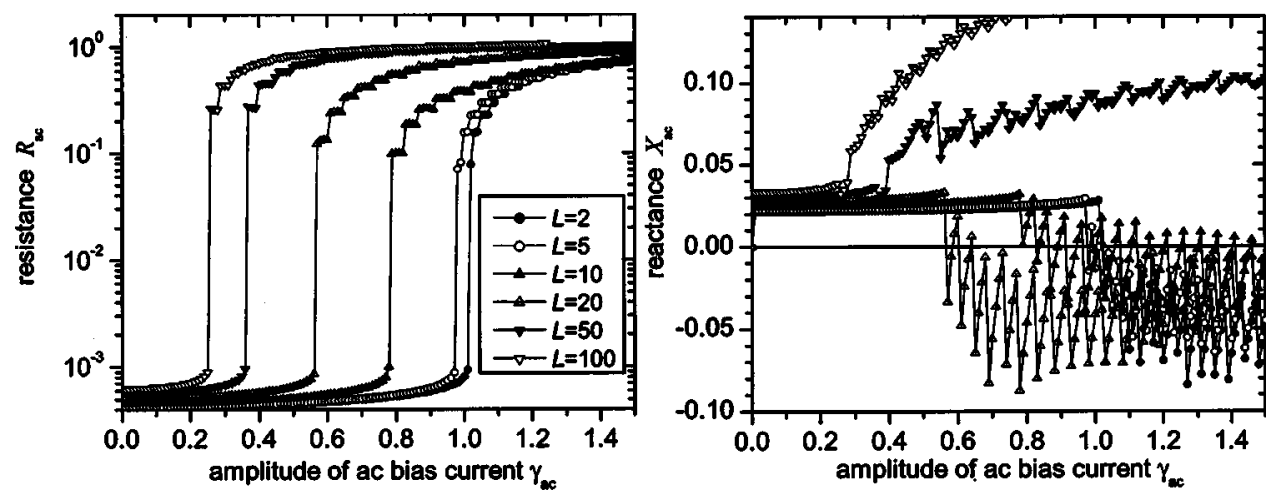

FIG. 2. The $R\left(\gamma_{\mathrm{ac}}\right)$ and $X\left(\gamma_{\mathrm{ac}}\right)$ dependencies for different LJJ length $L=2$, $5,10,20,50$, and 100 . 

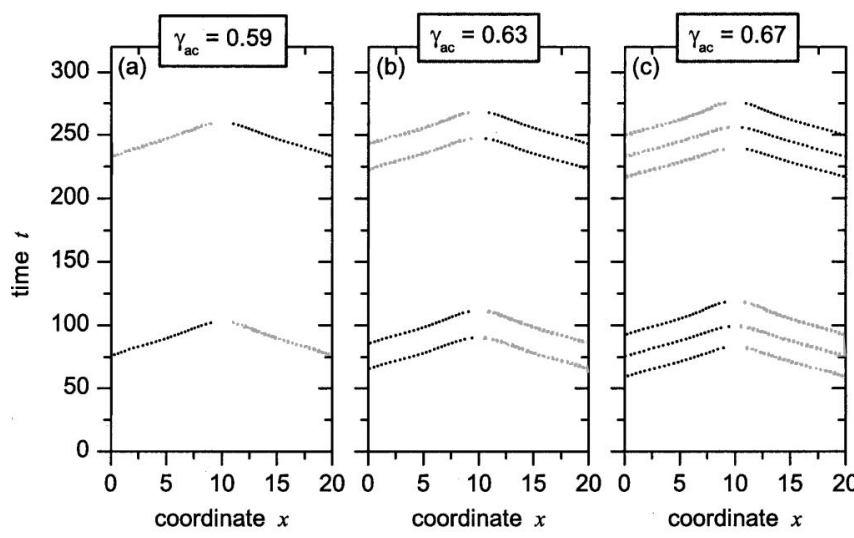

havior for large and small $L$. For not very long junction ( $L$ $\leqslant 20)$ and $\gamma_{\mathrm{ac}}>\gamma_{\mathrm{ac}}^{p}, X\left(\gamma_{\mathrm{ac}}\right)$ is saw-toothlike and takes negative as well as positive values. For $L \geqslant 50$ the dependence $X\left(\gamma_{\mathrm{ac}}\right)$ shifts to the region of positive values (inductive behavior) and has a more complex saw-toothlike structure.

To illustrate the difference between the junctions of moderate length and the very long ones, in Figs. 3 and 4 we present the fluxon's trajectories on the $x-t$ plane for $L$ $=20$ and $L=50$, respectively. For $L=20$, Fig. 3 shows the trajectories for different values of $\gamma_{\mathrm{ac}}$ corresponding to the first, the second, the third, and the sixth steps in the $R\left(\gamma_{\mathrm{ac}}\right)$ dependence. One can see that in the system biased at the first $R\left(\gamma_{\mathrm{ac}}\right)$ step, one $\mathrm{F}-\mathrm{AF}$ pair enters the junction during the positive semiperiod of the ac current. The instant value of $\sin (\omega t)$ is shown in the rightmost plot. Upon entrance, the fluxon (shown by the black color) and the antifluxon (shown by the gray color) are driven towards the center of the junction under the action of a Lorentz force created by the bias current. Since the LJJ is not extremely long, during the positive semiperiod of ac current, the fluxon and antifluxon collide and annihilate in the center of the junction. At higher values of $\gamma_{\mathrm{ac}}$, two, three, etc. F-AF pairs enter the junction, move towards the center, and annihilate as shown in Figs. $3(\mathrm{~b})-3(\mathrm{~d})$, and the number of F-AF pairs penetrated into the LJJ exactly corresponds to the step number on the $R\left(\gamma_{\mathrm{ac}}\right)$ dependence. Thus, each F-AF pair penetrated into the LJJ adds an additional quantum of dissipation.

For a rather long junction of $L=50$, the fluxon trajectories look qualitatively different. After entering at $\gamma_{\mathrm{ac}}>\gamma_{\mathrm{ac}}^{p}$ $\approx 0.35$, fluxons and antifluxons need more time to pass the

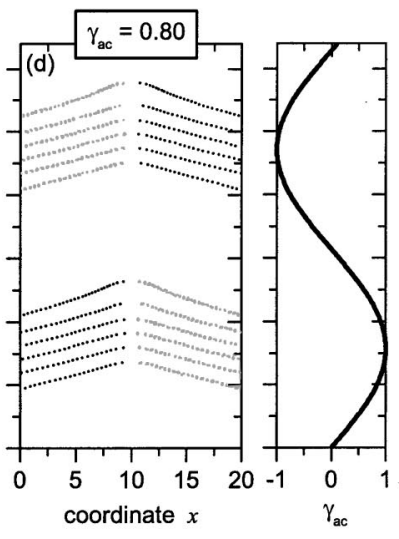

FIG. 3. Trajectories of fluxons for $L=20$ and $\alpha=1.0$. distance $L / 2$ and annihilate. If this does not happen during the positive semiperiod of ac current, the Lorentz force of the negative semiperiod pushes the fluxons and antifluxons back towards the edges of the LJJ. The corresponding fluxon trajectories are shown in Figs. 4(a) and 4(b) and correspond to the first and the fourth steps of the $R\left(\gamma_{\mathrm{ac}}\right)$ dependence. Note, that in the situation corresponding to Fig. 4(b), the fluxons leaving the LJJ during the negative semiperiod of the ac current collide with antifluxons which are entering the junction. In Fig. 4(b) three of four fluxons annihilate upon exit. This is a natural consequence of the time lag between the fluxon motion and the phase of ac current, which is roughly proportional to the length of the LJJ.

Further increase of the ac current in the resonator $\gamma_{\mathrm{ac}}$ results not only in a larger number of injected fluxons but also in a faster fluxon motion due to a stronger Lorentz force. In the overdamped case $(\alpha \geqslant 1)$ which we have considered up to now, the fluxon velocity is $\propto \gamma_{\mathrm{ac}}$. This means that at some value of $\gamma_{\mathrm{ac}}$ the velocity of fluxons becomes large enough for fluxons and antifluxons to pass the distance $L / 2$ during the semiperiod of ac current and annihilate in the center. The picture of fluxon trajectories, which corresponds to this situation is shown in Fig. 4(c). Here 5 fluxons and 5 antifluxons are injected into the junction, but only one $\mathrm{F}-\mathrm{AF}$ pair meets in the center of LJJ and annihilates. For even larger $\gamma_{\mathrm{ac}}$, a larger number of fluxons enters, but also a larger number of fluxons annihilates in the center.

The fluxon dynamics picture can be characterized by two numbers: $n_{\text {in }}$ is the number of injected F-AF pairs, and $n_{\text {an }}$ is the number of $\mathrm{F}-\mathrm{AF}$ pairs which meet and annihilate in the
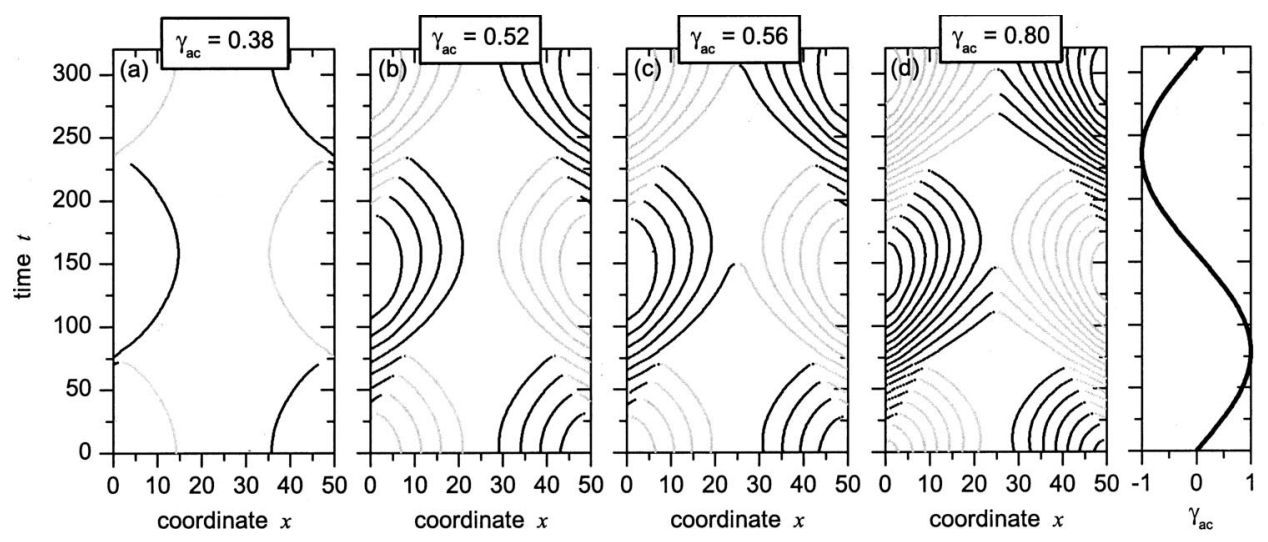

FIG. 4. Trajectories of fluxons for $L=50$ and $\alpha=1.0$. 

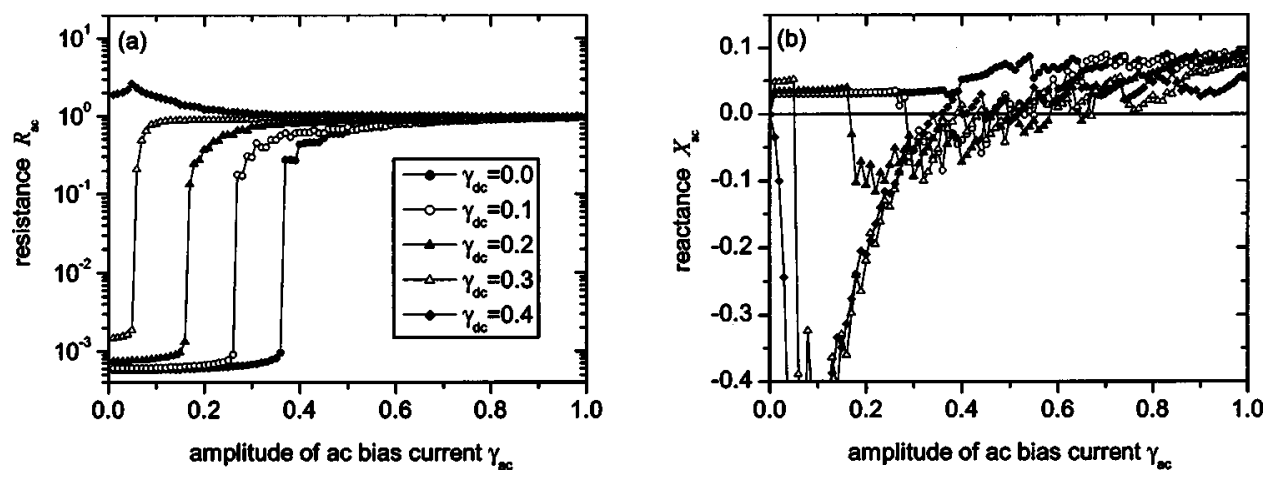

FIG. 5. The $R\left(\gamma_{\mathrm{ac}}\right)$ and $X\left(\gamma_{\mathrm{ac}}\right)$ dependencies for different values of dc bias current.

center of the LJJ. This classification is important because it corresponds to the different dissipation mechanisms present in the system. First, dissipation takes place due to the friction force. The energy dissipated in this way is proportional to the length of the path that the fluxon passes in the LJJ. Second, the dissipation is caused by annihilation of $\mathrm{F}-\mathrm{AF}$ pairs. Let us roughly evaluate the difference in the dissipated power (energy per semiperiod of ac current per $\mathrm{F}-\mathrm{AF}$ pair) in two different cases: (a) $n_{\text {in }}=5, n_{\text {an }}=0$ and (b) $n_{\text {in }}=5, n_{\text {an }}=1$. The difference in dissipated energy is

$$
\left(2 F_{\alpha} l+2 E_{\text {an }}\right)-\left(2 F_{\alpha} l / 2+E_{\text {an }}\right)=F_{\alpha} l+E_{\text {an }},
$$

where $F_{\alpha}$ is the average friction force acting on a fluxon and $E_{\text {an }}$ is the energy dissipated during annihilation. Thus, instead of making two full paths of length $l$ and two annihilations on exit, the $\mathrm{F}-\mathrm{AF}$ pair makes two half-paths and one annihilation in the center. This means that in the state $\left(n_{\mathrm{in}}, n_{\mathrm{an}}+1\right)$ the dissipation and, therefore $R\left(\gamma_{\mathrm{ac}}\right)$ is lower than in the state $\left(n_{\mathrm{in}}, n_{\mathrm{an}}\right)$. Therefore, in the $R\left(\gamma_{\mathrm{ac}}\right)$ dependence we should see not only steps up but also steps down upon increasing $\gamma_{\mathrm{ac}}$. If the system switches from the state $\left(n_{\text {in }}, n_{\text {an }}\right)$ to $\left(n_{\text {in }}, n_{\text {an }}+1\right)$, we should see a step down. If it switches to $\left(n_{\text {in }}+1, n_{\text {an }}\right)$ or $\left(n_{\text {in }}+1, n_{\text {an }}+1\right)$, we get a step up. Such steps are not easily visible in Fig. 2 for $L=50$, but more accurate inspection of the curve in the region corresponding to $n_{\mathrm{in}}=4, \ldots, 10$ shows that the steps down indeed exist, e.g., for $\gamma_{\mathrm{ac}}=0.75$ or $\gamma_{\mathrm{ac}}=0.93$. Very often the steps down are not observable because the system switches from the state $\left(n_{\text {in }}, n_{\text {an }}\right)$ directly to the state $\left(n_{\text {in }}+1, n_{\text {an }}+1\right)$.

Thus, LJJs of different length behave qualitatively differently and one can observe a crossover from moderate to long junction behavior related to the commensurability of the ac current period and the time $L / u$ which fluxon needs to pass the junction. In a junction of moderate length, two dissipation mechanisms are strongly coupled, so that $n_{\mathrm{in}}=n_{\text {an }}$. In a long junction these two mechanisms are to some extent independent, which may result in a nonmonotonic dependence of $R\left(\gamma_{\mathrm{ac}}\right)$.

\section{C. dc bias current}

Now we discuss how the fluxon dynamics picture and the measurable parameters such as $R\left(\gamma_{\mathrm{ac}}\right)$ and $X\left(\gamma_{\mathrm{ac}}\right)$ change, if an additional dc bias current $\gamma_{\mathrm{dc}}$ is injected into the LJJ. This problem is worth investigating because a dc bias current may provide the possibility of tuning a resonator.
The family of $R\left(\gamma_{\mathrm{ac}}\right)$ and $X\left(\gamma_{\mathrm{ac}}\right)$ dependencies for different values of $\gamma_{\mathrm{dc}}$ are shown in Figs. 5(a) and 5(b), respectively. Naturally, the injection of additional dc current $\gamma_{\mathrm{dc}}$ shifts all $R\left(\gamma_{\mathrm{ac}}\right)$ dependencies towards lower values of $\gamma_{\mathrm{ac}}$ by an amount which is approximately equal to $\gamma_{\mathrm{dc}}$. At the same time, the $X\left(\gamma_{\mathrm{ac}}\right)$ dependencies are not just shifted but also change their shape in quite a complex way. To understand how the dc current affects the fluxon dynamics inside the LJJ, in Figs. 6, 7, and 8 we present three families of fluxon trajectories for $\gamma_{\mathrm{dc}}=0.1,0.3$, and $\gamma_{\mathrm{dc}}=0.4$.

To see the effect of dc bias current $\gamma_{\mathrm{dc}}$, we have to compare these trajectories with the ones shown in Fig. 4. First, let us consider the effect of small dc bias current (see Fig. 6). When the first $\mathrm{F}-\mathrm{AF}$ pair penetrates into the LJJ at $\gamma_{\mathrm{ac}}=0.28$ [the first step on $R\left(\gamma_{\mathrm{ac}}\right)$ dependence], the $\mathrm{F}-\mathrm{AF}$ pair enters the LJJ at $t \approx 75$, first, approaching each other, and then, turning back when the negative period starts, very similar to the case $\gamma_{\mathrm{dc}}=0$ shown in Fig. 4(a). But in our case, the dc bias current $\gamma_{\mathrm{dc}}=0.1$ creates an additional driving force which pushes the fluxon to the left and the antifluxon to the right, i.e., towards each other. This finally results in the collision and annihilation of the fluxon and the antifluxon at $t$ $\approx 80$ during the next period. Note also, that $\gamma_{\mathrm{dc}}>0$ "helps" to inject the $\mathrm{F}-\mathrm{AF}$ pairs during the positive semiperiod, but hamper during the negative semiperiod. In fact, in Fig. 6(a) no fluxons are injected during the negative semiperiod.

The fluxon trajectories corresponding to the second step are shown in Fig. 6(b). Here two F-AF pairs are injected into the system. The first one annihilates after making a characteristic long loop like in the previous case, and another pair leaves the LJJ as in Fig. 4(a). Figure 6(c) shows the trajectories for the third step on $R\left(\gamma_{\mathrm{ac}}\right)$, where we see that two $\mathrm{F}-\mathrm{AF}$ pairs out of three make a long loop and one enters and exits similar to Fig. 4(a). Three different kinds of behavior can be seen in Fig. 6(d) corresponding to $\gamma_{\mathrm{ac}}=0.32$. Here, the driving ac current is strong enough to make the first $\mathrm{F}-\mathrm{AF}$ pair meet in the center. The second $\mathrm{F}-\mathrm{AF}$ pair passes a long loop path and finally collides under the action of $\gamma_{\mathrm{dc}}$. The third F-AF pair enters and exits as in the case $\gamma_{\mathrm{dc}}=0$.

The dynamics of fluxons corresponding to higher steps is shown in Fig. 6(e)-6(h). We see that the long loops may appear and disappear as $\gamma_{\mathrm{ac}}$ grows. They represent an intermediate trajectory between the situation where a F-AF pair enters and then exists, and the situation where a F-AF pair enters and annihilates in the center of the LJJ. Note, that at $\gamma_{\mathrm{ac}}=0.48$ the fluxons which are about to leave the junction 

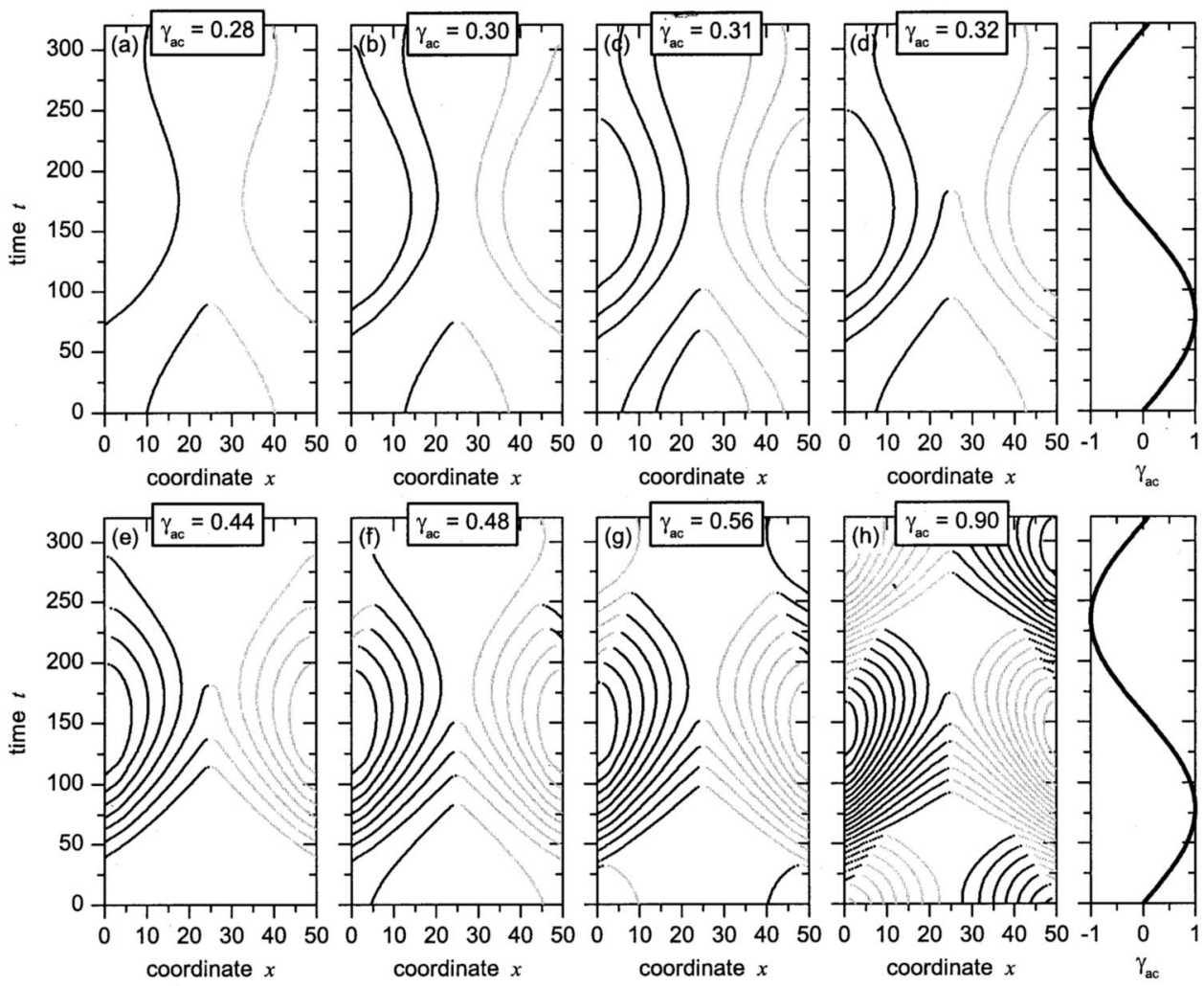

FIG. 6. Trajectories of fluxons for $L=50, \alpha=1.0$, and $\gamma_{\mathrm{dc}}=0.1$.

collide with antifluxons which are entering the LJJ during the negative semiperiod, thus producing an additional dissipation. This picture is similar to Figs. 4(c) and 4(d).

In Fig. 6(g) one can see the first $\mathrm{F}-\mathrm{AF}$ pair enters the LJJ during the negative semiperiod without annihilation with $\mathrm{F}-\mathrm{AF}$ pairs of the positive semiperiod. At even higher bias current, see, e.g., Fig. 6(h), the number of F-AF pairs which enter the LJJ during both positive and negative semiperiod grows but the asymmetry introduced by $\gamma_{\mathrm{dc}} \neq 0$ still remains visible, e.g., compare the number of $\mathrm{F}-\mathrm{AF}$ pairs which enter during positive $n_{\text {in }}^{+}$and negative $n_{\text {in }}^{-}$semiperiods. As $\gamma_{\text {ac }}$ grows further, the picture becomes more and more symmetric. This is quite natural since $\gamma_{\mathrm{dc}}$, which we keep at constant level, becomes negligible in comparison with $\gamma_{\mathrm{ac}}$.

With the penetration of the first $\mathrm{F}-\mathrm{AF}$ pair during the negative semiperiod, the long looplike trajectories do not appear anymore. Note, that they may originate only from the $\mathrm{F}-\mathrm{AF}$ pairs of the positive semiperiod. For $\mathrm{F}-\mathrm{AF}$ pairs of the negative semiperiod the current $\gamma_{\mathrm{dc}}$ creates only a repelling force, so that long looplike trajectories are impossible.

We can already see that the fluxon dynamics here may be characterized by five numbers: $n_{\text {in }}^{+}$and $n_{\text {in }}^{-}$are the numbers of $\mathrm{F}-\mathrm{AF}$ pairs which enter during the positive and the negative semiperiod, respectively, $n_{\text {an }}^{+}$and $n_{\text {an }}^{-}$are the numbers of $\mathrm{F}-\mathrm{AF}$ pairs which are annihilated in the center of the junction during the positive and the negative semiperiod, respectively, and $n_{\text {an }}^{*}$ is the number of F-AF pairs which are annihilated in the center after passing a long looplike trajectory. Such F-AF pairs can enter the LJJ only during the positive semiperiod for $\gamma_{\mathrm{dc}}>0$ and only during negative semiperiod for $\gamma_{\mathrm{dc}}<0$.

On the base of these numbers, we can evaluate the energy dissipated per ac period in different configurations. We leave the complete analysis to the reader (it is straightforward), but show again one example which demonstrates an-

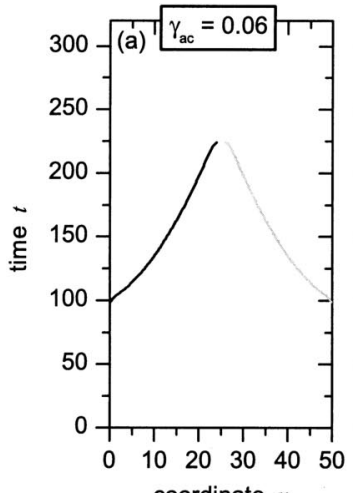

coordinate $x$

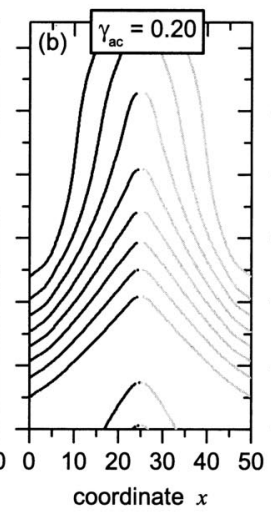

coordinate $x$

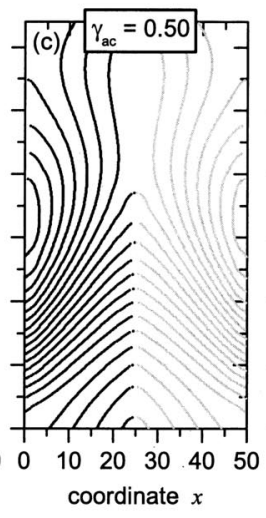

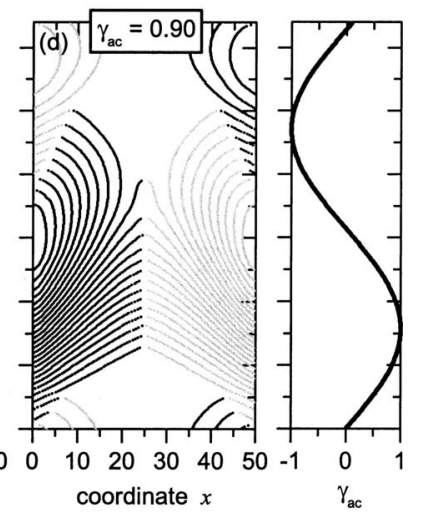

FIG. 7. Trajectories of fluxons for $L=50, \alpha=1.0$, and $\gamma_{\mathrm{dc}}=0.3$. 

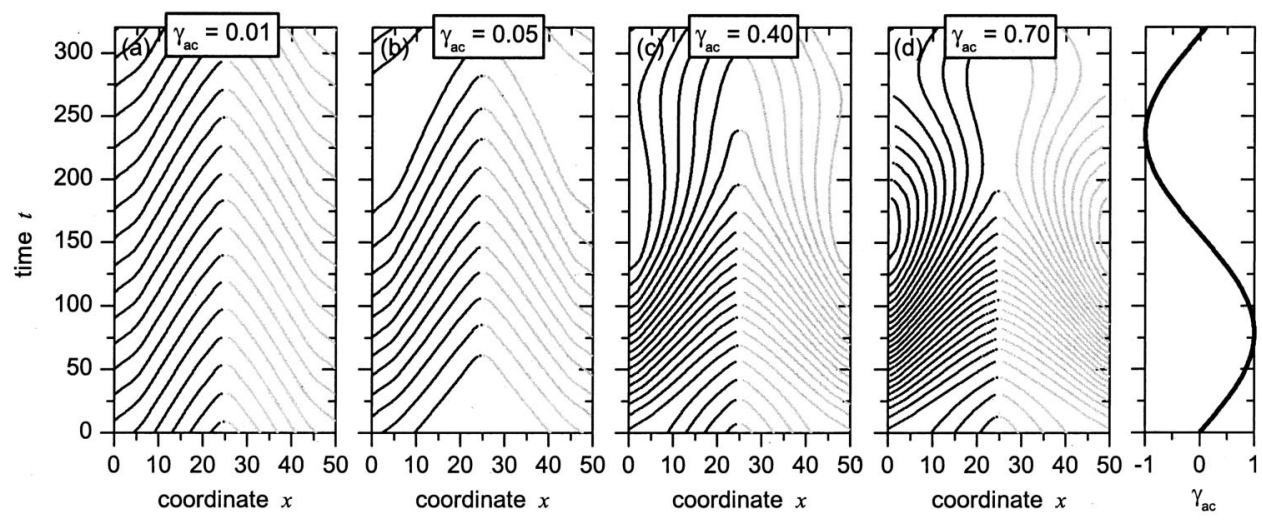

FIG. 8. Trajectories of fluxons for $L=50, \alpha=1.0$, and $\gamma_{\mathrm{dc}}=0.4$. other mechanism of the appearance of lower steps on the $R\left(\gamma_{\mathrm{ac}}\right)$ curve for higher $\gamma_{\mathrm{ac}}$. Compare two plots in Figs. 6(c) and $6(\mathrm{~d})$. For $\gamma_{\mathrm{ac}}=0.31$ we have $n_{\mathrm{in}}^{+}=3, n_{\mathrm{an}}^{+}=0$, and $n_{\mathrm{an}}^{*}$ $=2$, while for $\gamma_{\mathrm{ac}}=0.32$ we have $n_{\mathrm{in}}^{+}=3, n_{\text {an }}^{+}=1$, and $n_{\mathrm{an}}^{*}$ $=1$, (i.e., one $\mathrm{F}-\mathrm{AF}$ pair instead of making a long loop and annihilating, annihilates right in the center). Thus the system does not dissipate the energy $F_{\alpha}\left(l_{\mathrm{lp}}-l_{\mathrm{sp}}\right)$, where $l_{\mathrm{lp}}$ and $l_{\mathrm{sp}}$ are the lengths of the long and short paths, accordingly. So, here we see another mechanism which leads to, in some extent, a counterintuitive result: we pump more power into the resonator, but losses drop.

The sequence of trajectories shown in Fig. 6 is valid only for small values of $\gamma_{\mathrm{dc}}$. At higher values, e.g., $\gamma_{\mathrm{dc}}$ $=0.3$ shown in Fig. 7 , the first $\mathrm{F}-\mathrm{AF}$ pair which penetrates into LJJ does not make a long loop as in Fig. 6(a), but rather annihilates in the center very soon [see Fig. 7(a)]. Further increase of the $\gamma_{\mathrm{ac}}$ results in the injection of additional $\mathrm{F}-\mathrm{AF}$ pairs into the LJJ during the positive semiperiod, but all of them correspond to the case $n_{\text {in }}^{+}=n_{\text {an }}^{+}$. At higher $\gamma_{\text {ac }}$ the trajectories corresponding to the few last $\mathrm{F}-\mathrm{AF}$ pairs smoothly transform into the long looplike trajectories, as can be seen in Fig. 7(b). Then, at even higher value of $\gamma_{a c}$, we can have all three different kinds of trajectories as shown in Fig. 7(c), but still no $\mathrm{F}-\mathrm{AF}$ injected during the negative semiperiod. The $\gamma_{\mathrm{ac}}=0.70$ (not shown) is large enough also to inject the $\mathrm{F}-\mathrm{AF}$ pairs during the negative semiperiod. At the beginning they immediately annihilate with antisolitons which were injected during the positive semiperiod and are about to leave the LJJ. But at a little bit higher value of $\gamma_{\mathrm{ac}}$, e.g., $\gamma_{\mathrm{ac}}$ $=0.90$ as in Fig. 7(d), the F-AF pairs injected during the negative semiperiod do not only annihilate but some of them can move in the junction. Note, that these F-AF pairs annihilate with an $\mathrm{F}-\mathrm{AF}$ of the next positive semiperiod just before exiting the LJJ.

In the case when $\gamma_{\mathrm{dc}}$ exceeds some critical value, the system always has rather high losses as can be seen in Fig. 5(a) for $\gamma_{\mathrm{dc}}=0.4$. In this case, even for very small or zero $\gamma_{\mathrm{ac}}$, corresponding to the resonator which is switched off, we observe the fluxon trajectories which are shown in Fig. 8(a). We see that even at low ac bias current the F-AF pairs enter the LJJ from the left and right edge and annihilate in the center. This happens only due to the nonuniform current distribution (2) which "automatically" injects fluxons and antifluxons through the edges and provides both channels of dissipation: due to friction and due to annihilation. When we excite the small amplitude oscillations in the resonator the permanent injection of $\mathrm{F}-\mathrm{AF}$ pairs is suspended for some time during the negative semiperiod at the moment when the ac bias has its minimum which counteracts against $\gamma_{\mathrm{dc}}$ as can be seen in Fig. 8(b). Further increase of $\gamma_{a c}$ results in the $\mathrm{F}-\mathrm{AF}$ pair injection only during the positive semiperiod and transforms the trajectories of the few last $\mathrm{F}-\mathrm{AF}$ pairs to the long looplike trajectories as shown in Fig. 8(c). Three different kinds of trajectories can be observed in Fig. 8(d). At higher values of $\gamma_{\mathrm{ac}}$, the picture of fluxon trajectories evolves very similar to the two previous cases of $\gamma_{\mathrm{dc}}=0.1$ and 0.3 .

\section{D. dc magnetic field}

Now let us investigate the effect of the applied magnetic field on the properties of the system and, in particular, on the fluxon dynamics in the LJJ. The magnetic field is applied perpendicular to the plane of the structure. For planar geometry, as in the case of GB LJJ, the direction of the external magnetic field coincides with the direction of the magnetic field of vortices that can be located inside the LJJ. Due to the Meissner effect the magnetic field is expelled from the film and has a larger amplitude at the edges (so-called flux focusing effect), in particular at $x=0$ and $x=L$. We do not consider the details related to this effect here and assume that the amplitude of external magnetic field is specified taking into account the flux focusing. If we speak about overlap geometry, then the direction of magnetic field is parallel to the plane of the sample and perpendicular to the $x$ direction of the LJJ.

The families of $R\left(\gamma_{\mathrm{ac}}\right)$ and $X\left(\gamma_{\mathrm{ac}}\right)$ dependencies calculated for different values of the applied magnetic field $h$ are shown in Figs. 9(a) and 9(b), respectively. The $R\left(\gamma_{\mathrm{ac}}\right)$ has a similar steplike behavior, but the value of losses is, in general, rather high even at very small power levels. For $h=1$ the $R\left(\gamma_{\mathrm{ac}}\right)$ curve first shows a decrease of losses (several steps downwards) and then an increase (steps upwards), which looks quite unusual and counterintuitive; the losses decrease as we increase the amplitude of oscillations in the resonator. Although a similar result was obtained above for the case $\gamma_{\mathrm{ac}} \neq 0$, here it is not just a small single step but a series of such steps.

To understand the mechanism of this phenomenon in detail, we calculate the fluxon trajectories corresponding to 

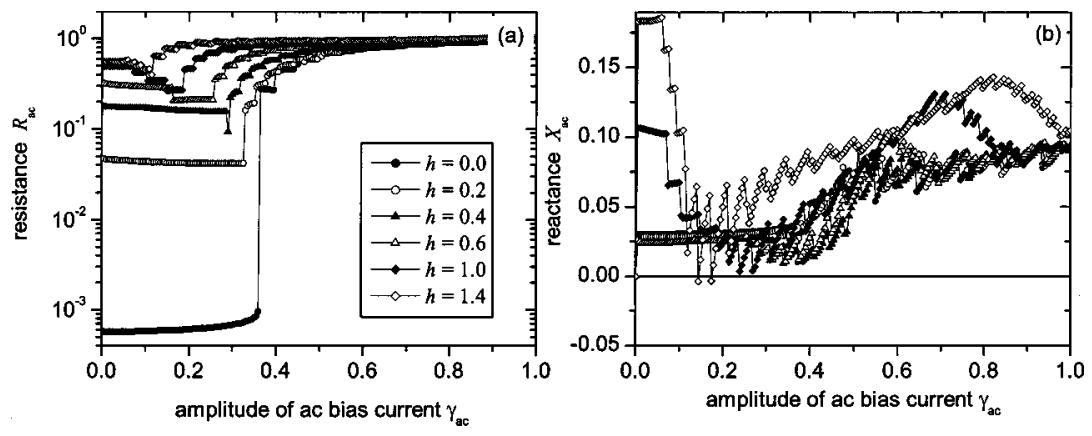

FIG. 9. The $R\left(\gamma_{\mathrm{ac}}\right)$ and $X\left(\gamma_{\mathrm{ac}}\right)$ dependencies for different values of externally applied magnetic field. different values of $h$ and $\gamma_{\mathrm{ac}}$. A set of fluxon trajectories for $h=0.2$ is shown in Fig. 10. Let us again compare them with Fig. 4. The substantial losses at small values of $\gamma_{\mathrm{ac}}$ are related to the presence of a fluxon in the LJJ. As initial conditions we have used a linear phase distribution $\phi(x)=h x$, which is rather realistic assumption (see below). The vortex almost does not move since the applied bias current $\gamma_{\mathrm{ac}}$ is very small. As the power in the resonator increases up to the precritical value $\gamma_{\mathrm{ac}}=0.32$, the fluxon oscillates back and forth inside the LJJ as shown in Fig. 10(b). The substantial dissipation is provided by the fluxon always present in the LJJ. Note that there are no antifluxons entering the junction in this mode, which is quite natural since the external magnetic field tries to penetrate into the LJJ creating a positive nonzero fluxon density, i.e., favoring the fluxons of the positive polarity only.

When the bias point moves to the first step of the $R\left(\gamma_{\mathrm{ac}}\right)$ dependence, the losses increase because of the penetration of an additional fluxon into the LJJ as shown in Fig. 10(c) corresponding to $\gamma_{\mathrm{ac}}=0.34$. Note that the fluxon is not trapped in the system but enters and leaves the junction twice during each ac period. During the positive semiperiod the fluxon exits through the right edge and, simultaneously, another fluxon enters through the left edge. During the negative semiperiod the fluxon exits through the left edge and, at the same time, another fluxon enters through the right edge. The joint action of the ac current and external field creates a larger effective field at the edge of the junction where the fluxon is injected. At the other edge the effective field is smaller, so that the antifluxons are not injected. During the negative semiperiod the picture is similar but the roles of the edges exchange. The presence (in average) of an additional fluxon results in an additional channel of dissipation due to friction.

With the further increase of $\gamma_{\mathrm{ac}}$ the bias point passes several steps along the $R\left(\gamma_{\mathrm{ac}}\right)$ dependence. Each of these steps corresponds to different numbers of additionally injected fluxons $n_{\text {in }}^{+}$, while the initially trapped fluxon still stays trapped in the system, e.g., as shown in Fig. 10(d)
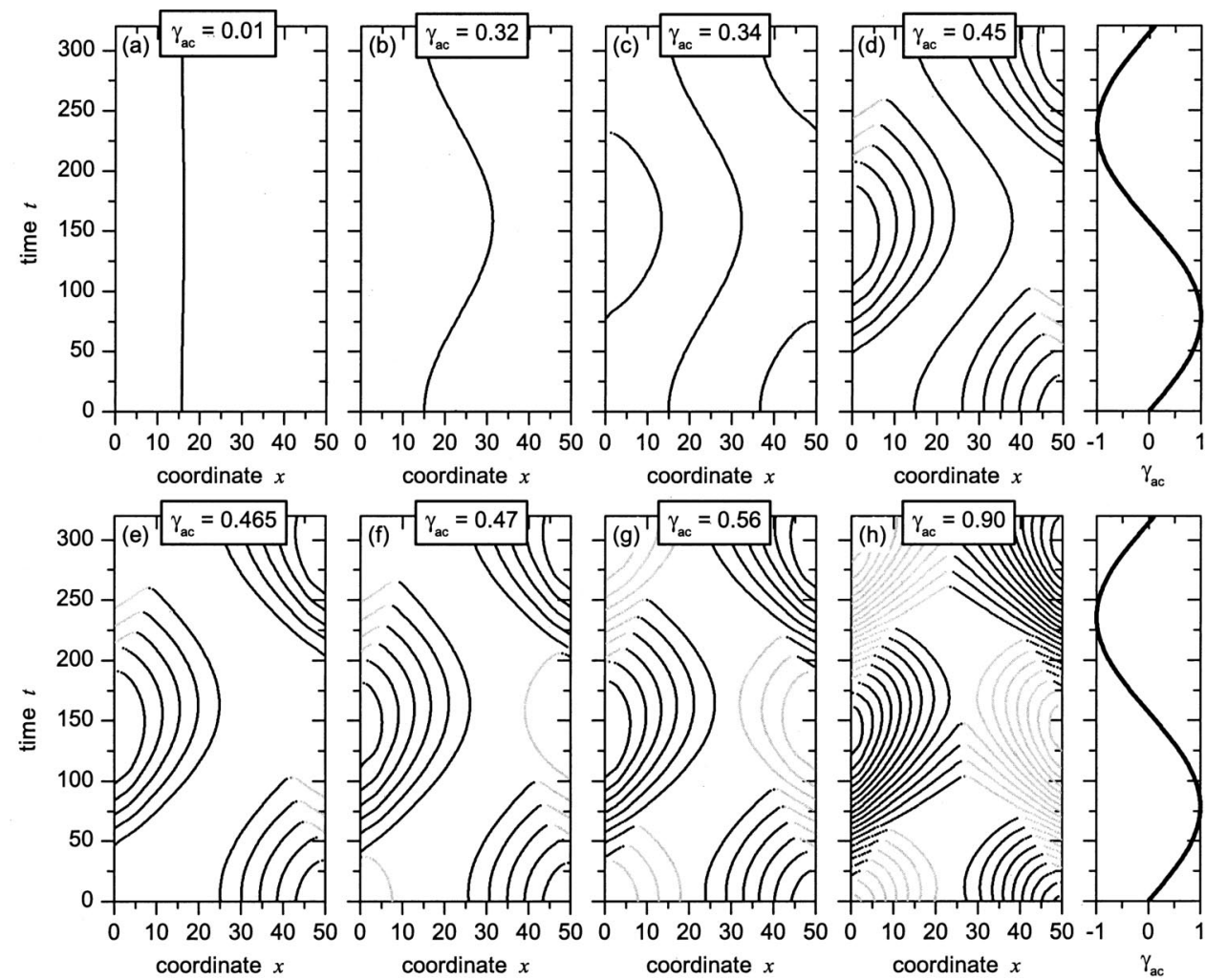
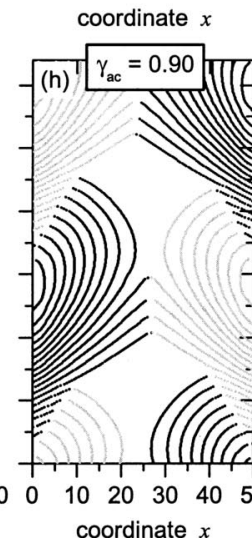

$\begin{array}{lll}1020 & 30 \quad 40 \\ \text { coordinate } x\end{array}$

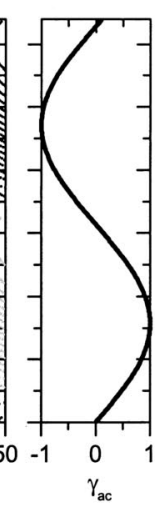

FIG. 10. Trajectories of fluxons for $L=50, \alpha=1.0$, and $h=0.2$. 

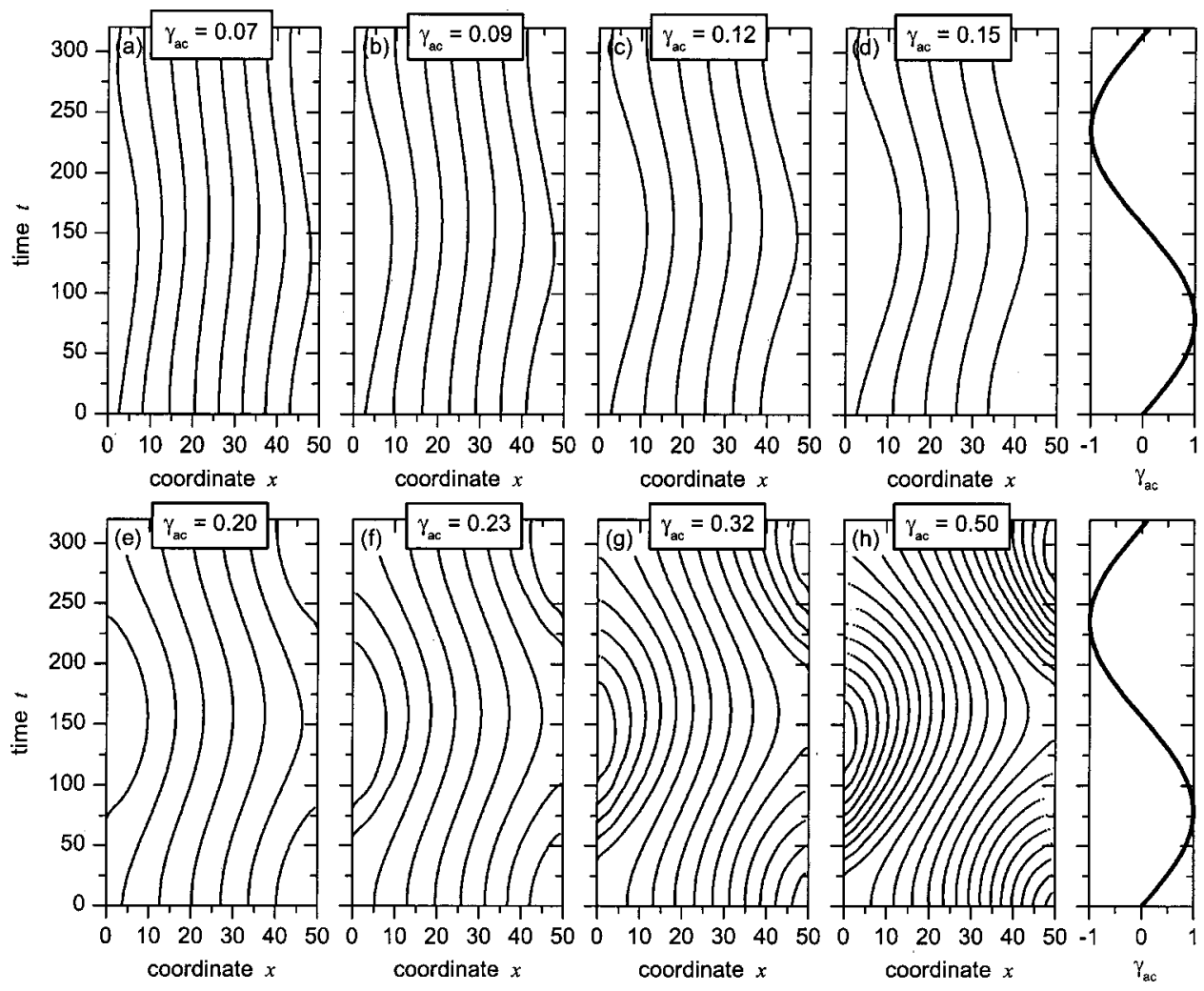

FIG. 11. Trajectories of fluxons for $L=50, \alpha=1.0$, and $h=1.0$.

corresponding to the fifth step at $\gamma_{\mathrm{ac}}=0.45$. One may note that during the positive and negative semiperiods the antifluxons are injected in the junction, but they immediately annihilate with fluxons which are about to leave the junction through the same edge. Thus, the second mechanism of losses, $\mathrm{F}-\mathrm{AF}$ annihilation, starts to contribute to the net dissipated energy.

Up to now the increase of $\gamma_{\mathrm{ac}}$ has not only resulted in injection of fluxons but also in increasing the oscillation amplitude of the trapped fluxon. At $\gamma_{\mathrm{ac}}=0.465$ this amplitude becomes so large that the trapped fluxon leaves the system as shown in Fig. 10(e). It is quite obvious that the dynamical fluxon state shown in Fig. 10(e) dissipates less energy than the state shown in Fig. 10(d), by the amount which the trapped fluxon has dissipated due to the friction. This effect manifests itself as a step downwards on the $R\left(\gamma_{\mathrm{ac}}\right)$ dependence at $\gamma_{\mathrm{ac}}=0.465$, which can be seen in Fig. 9(a).

Figure 10(f) shows that at $\gamma_{\mathrm{ac}}=0.47$ the first antifluxon enters the LJJ and moves during substantial time without annihilation. In fact annihilation takes place only when the antifluxon is about to leave the junction. Further pumping of the resonator results in larger number of $\mathrm{F}-\mathrm{AF}$ pairs entering the LJJ as shown in Fig. 10(g) and, eventually, in annihilation between some of the F-AF pairs in the center of the LJJ as shown in Fig. 10(h). In the limit of a very strong ac drive, when the equivalent magnetic field created by the ac bias current is much larger than the external magnetic field, the fluxon dynamics picture becomes more symmetric and is reminiscent of the one shown in Fig. 4(d).

For larger values of the external magnetic field, the $R\left(\gamma_{\mathrm{ac}}\right)$ exhibits nonmonotonic behavior. A set of fluxon trajectories is shown in Fig. 11. At low $\gamma_{\mathrm{ac}}$ there are 8 trapped fluxons that oscillate under the action of $\gamma_{\mathrm{ac}}$. The dissipation is about $8 F_{\alpha} l$. With increasing $\gamma_{\mathrm{ac}}$ the amplitude of these oscillations also grows and, at some point, one of the fluxons is kicked out of the junction, as shown in Fig. 11(b). The dissipation decreases down to $7 F_{\alpha} l$. As the fluxon oscillation amplitude grows further, the system gets rid of fluxons one by one as can be seen in Figs. 11(b)-11(d), which corresponds to the consecutive steps downwards in the $R\left(\gamma_{\mathrm{ac}}\right)$ dependence shown in Fig. 9(a).

Eventually the magnetic field generated by $\gamma_{\mathrm{ac}}$ together with the external magnetic field becomes large enough to inject the fluxon through the left edge of the LJJ during the positive semiperiod and through the right edge during the negative semiperiod, as shown in Fig. 11(e). The presence (in average) of one additional fluxon results in an increase of the friction force and, therefore, in a step with higher resistance in the $R\left(\gamma_{\mathrm{ac}}\right)$ dependence. Further increase of $\gamma_{\mathrm{ac}}$ results in the injection of 2, 3, etc., additional fluxons [see Figs. 11(f) $-11(\mathrm{~h})]$ and progressive steps with higher resistance in the $R\left(\gamma_{\mathrm{ac}}\right)$ dependence.

To describe the dynamic state we can introduce the following quantities: $n_{\mathrm{tr}}^{+}$and $n_{\mathrm{tr}}^{-}$are the numbers of trapped fluxons and antifluxons, $n_{\text {in }}^{+}$and $n_{\text {in }}^{-}$are the numbers of injected fluxons and antifluxons, respectively, and $n_{\text {an }}$ is the number of $\mathrm{F}-\mathrm{AF}$ pairs annihilated in the center of the LJJ as in Fig. 10(h). It is clear that switching from the state $\left(n_{\text {in }}, n_{\text {tr }}\right)$ to $\left(n_{\text {in }}, n_{\text {tr }}-1\right)$ or from $\left(n_{\text {in }}, n_{\text {an }}\right)$ to $\left(n_{\text {in }}, n_{\text {an }}+1\right)$ results in a lower dissipation.

Analyzing Figs. 9-11, one might notice that for $\gamma_{\mathrm{ac}}$ $\rightarrow 0$ the fluxons in the LJJ are present for a value of field $h<2$ ( 2 being the penetration field in normalized units). This 


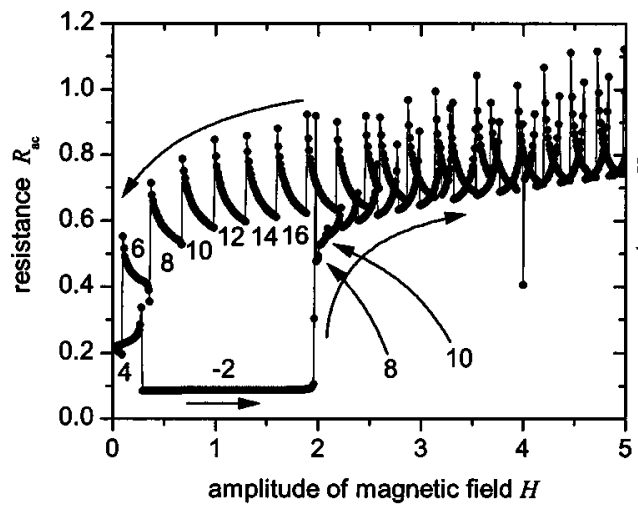

FIG. 12. The $R(h)$ and $X(h)$ dependencies for $\gamma_{\mathrm{ac}}=0.01, L=50$, and $\alpha=1.0$. happens because we have used the linear phase $\phi(x)=h x$ as the initial phase distribution. Such a phase distribution was chosen because once the fluxons penetrated into the LJJ, it could be very difficult to get rid of them. To illustrate this, we have simulated the impedances $R(h)$ and $X(h)$ of the system as a function of the applied magnetic field $h$ at the small value of $\gamma_{\mathrm{ac}}=0.01$. These dependencies are shown in Fig. 12. The field was swept from zero up to +7 , then down to -7 , then again up to +7 , etc. The initial state without trapped fluxons is visible clearly during the first sweep from 0 to 7 , but never appears again. Instead, the system always has some trapped fluxons or antifluxons even at zero applied magnetic field. In Fig. 12 we show only the positive part of the hysteretic loop, since the negative part is completely symmetric, i.e., $R(-h)=R(h)$ and $X(-h)=X(h)$. The numbers next to the sub-branches in Fig. 12 indicate the number of trapped fluxons (negative number indicate antifluxons). On the negative part the numbers change sign.

First, we see characteristic sub-branches (states) with different number of trapped fluxons. Second, the switching between neighboring states takes place when one pair of fluxons (or antifluxons) enters or leaves the LJJ. This happens in pairs because of the symmetry (in simulation) of the system relative to the injection of fluxons from the left and right edge. Third, the peaks of $R$ in each state correspond to the configuration where the outermost fluxons are extremely close to the junction edges. Higher ac current density at the edges moves these fluxons with much larger amplitude than all other fluxons resulting in a higher dissipation.

These peaks are very similar to the ones observed experimentally and calculated numerically in Ref. 6. The re- markable difference between our results and results obtained in Ref. 6 is that in our case the oscillations occur at the high average level of losses from $10^{-1}$ to 1 , while in Ref. 6 losses $(1 / Q)$ seem to change in the range from $10^{-4}$ to 1 . The authors of Ref. 6 explain the observed peaks as a manifestation of the individual fluxons entering the LJJ. Our understanding and the results of our simulations suggest that when fluxons enter the junction, they fill it quite uniformly with some nonzero average density, thus increasing the losses drastically in comparison with the empty junction. So, the losses increase and stay high. It is not clear to us how the system described in Ref. 6 recovers and switches back to the low losses regime, in which, presumably, there are no fluxons in the LJJ. In the experiment, this still may happen due to the presence of parasitic dc (or low frequency) bias currents, or noise, but in the simulations reported in Ref. 6 there should be another mechanism for this.

\section{E. Underdamped case}

To make the comparison between the overdamped ( $\alpha$ $\geqslant 1$ ) and the underdamped $(\alpha<1)$ cases easier, we present the numerical results for a weakly underdamped case junction with $\alpha=0.1, \ldots, 1.0$. The dependencies $R\left(\gamma_{\mathrm{ac}}\right)$ and $X\left(\gamma_{\mathrm{ac}}\right)$ calculated numerically for the different $\alpha$ and $L$ $=50$, are shown in Fig. 13. One can see that for a small current amplitude, when $\gamma_{\mathrm{ac}}<\gamma_{\mathrm{ac}}^{p} \approx 0.35$, the resistance is the lowest for the smallest damping $\alpha=0.1$, which is quite natural. Unexpectedly, for $\gamma_{\mathrm{ac}}>\gamma_{\mathrm{ac}}^{p}$, when the fluxons enter the $\mathrm{LJJ}$, the situation is opposite, i.e., for $\alpha_{1}<\alpha_{2}$ the resistance $R\left(\alpha_{1}\right)>R\left(\alpha_{2}\right)$ is at the same value of $\gamma_{\mathrm{ac}}$. The dependence
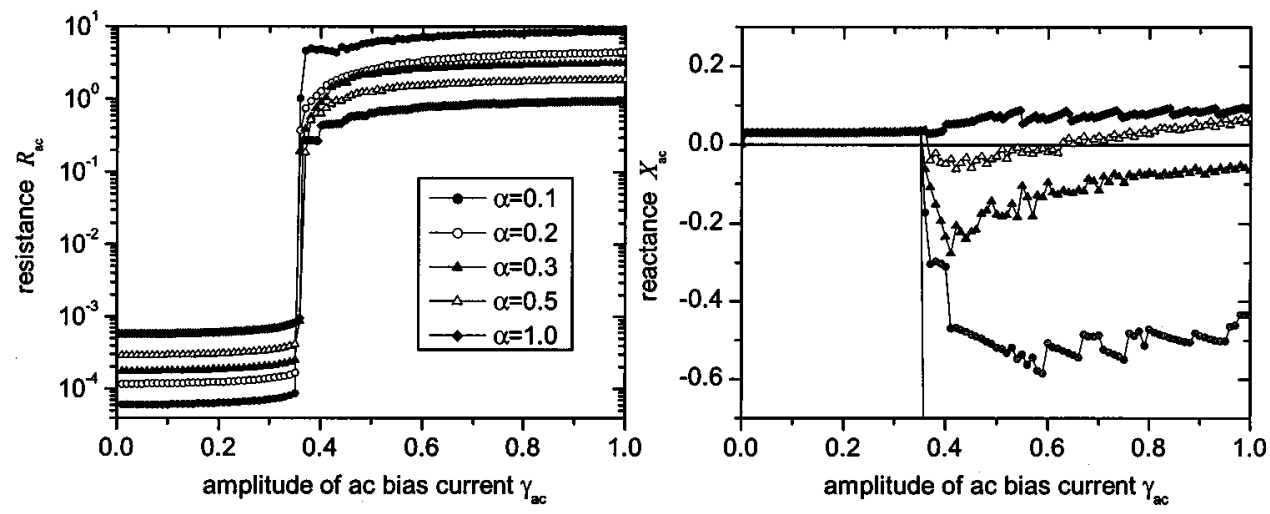

FIG. 13. The $R\left(\gamma_{\mathrm{ac}}\right)$ and $X\left(\gamma_{\mathrm{ac}}\right)$ dependencies for different values of $\alpha=0.1, \ldots, 1.0$ and $L=50$ 

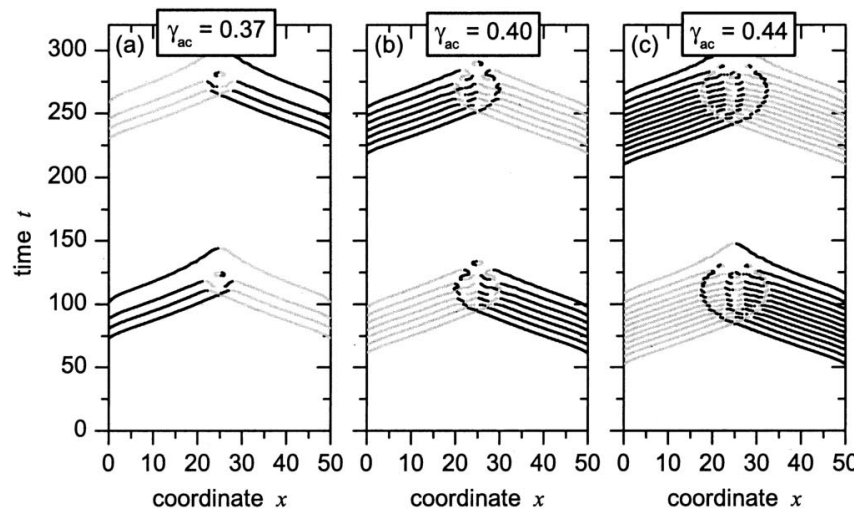

$X\left(\gamma_{\mathrm{ac}}\right)$ goes deep into the negative region as $\alpha$ decreases, which is the manifestation of the major role of capacitance in the case $\alpha<1$. The fluxon trajectories for the same parameters as in Fig. 4 and $\alpha=0.2$ are shown in Fig. 14 .

First, at such a low damping the F-AF pairs injected into the junction by the ac bias current move much faster, easily reaching velocities $\sim \bar{c}_{0}$. Therefore, it takes less time to reach the center of the junction and annihilate with antifluxon. The trajectories look almost like in the case $L=20$ for $\alpha=1$. In fact, when relativistic behavior starts to be involved we should revise our arguments about crossover between the moderate and very long JJ. For a F-AF pair to meet in the center, the length should satisfy the following expression $L \bar{u}>2 \pi / \omega$. If the fluxons move with relativistic velocities, the maximum velocity is no longer $\propto \gamma_{\mathrm{ac}}$, but saturates at $u=\bar{c}_{0}$, i.e., if the length $L>2 \pi / \omega \bar{c}_{0}$, the fluxon does not meet an antifluxon in the center of the junction even when they both move with the maximum possible velocity.

Second, the F-AF pairs do not obligatory annihilate when they collide as it happens for the overdamped case. Fluxon and antifluxon pass through each other losing some portion of kinetic energy, which is dissipated due to the presence of the $\alpha$ term in Eq. (1). The passage without annihilation usually takes place when $\mathrm{F}-\mathrm{AF}$ pairs collide at high relative velocity. It was found ${ }^{11}$ that when a $\mathrm{F}-\mathrm{AF}$ pair is driven by the uniform dc bias current $\gamma>4 \alpha^{3 / 2}$, it does not annihilate. The loss of kinetic energy results in a decrease of the velocity. After several collisions the velocity becomes low enough, and the fluxon annihilates during the collision with the next antifluxon, if any. This process can be seen in Fig. 14.

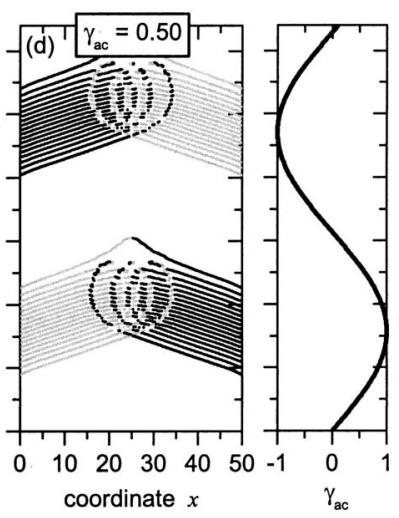

FIG. 14. The fluxons trajectories for $L=50$ and $\alpha=0.2$.
The fluxon trajectories can be more complex than the ones shown in Fig. 14. As an example, in Fig. 15 we show the trajectories for $L=20$ and $\alpha=0.2$. In Fig. 15(a) $\left[\gamma_{\mathrm{ac}}\right.$ $=0.56$, the first step on $\left.R\left(\gamma_{\mathrm{ac}}\right)\right]$ the fluxon and antifluxon collide in the center of the junction, pass through each other, collide with the opposite edge of the LJJ, reflect as solitons of opposite polarity, collide in the center again, and, finally, exit the LJJ through the edges. The result of the collision with the edges depends on the fluxon velocity and driving current. If they both are too low, the fluxons may not reflect. In our case, the driving current amplitude $\gamma_{\mathrm{ac}}$ is a function of time. At $t \approx 120$ the driving current is large enough and the solitons are reflected, but at $t \approx 155 \gamma_{\text {ac }} \sin (\omega t)$ is almost zero and reflection does not take place. At a slightly higher value of the power $\left[\gamma_{\mathrm{ac}}=0.57\right.$, Fig. $\left.15(\mathrm{~b})\right]$ the picture is more complex. Four F-AF pairs are injected into the LJJ, 4 fluxons and 4 antifluxons pass through each other and are reflected from the opposite edges of the LJJ. Note that the "beams" of fluxons get larger after "scattering" on each other. On the way back, all four F-AF pairs annihilate being unsupported by the ac bias current $\gamma_{\mathrm{ac}} \sin (\omega t)$ which at this moment changes the sign from positive to negative.

At $\gamma_{\mathrm{ac}}=0.58$ the picture shown in Fig. 15(c) is even more complex. The processes which take place during the positive and negative semiperiod are not the same. After the initial collision of the chain of fluxons (moving from the left to the right) with the chain of antifluxons (moving from the right to the left), and after reflection of both chains from the opposite edges of the junction, all F-AF pairs annihilate except one. Fluxon and antifluxon of the left pair exit the collision region and move inside the junction reflecting several
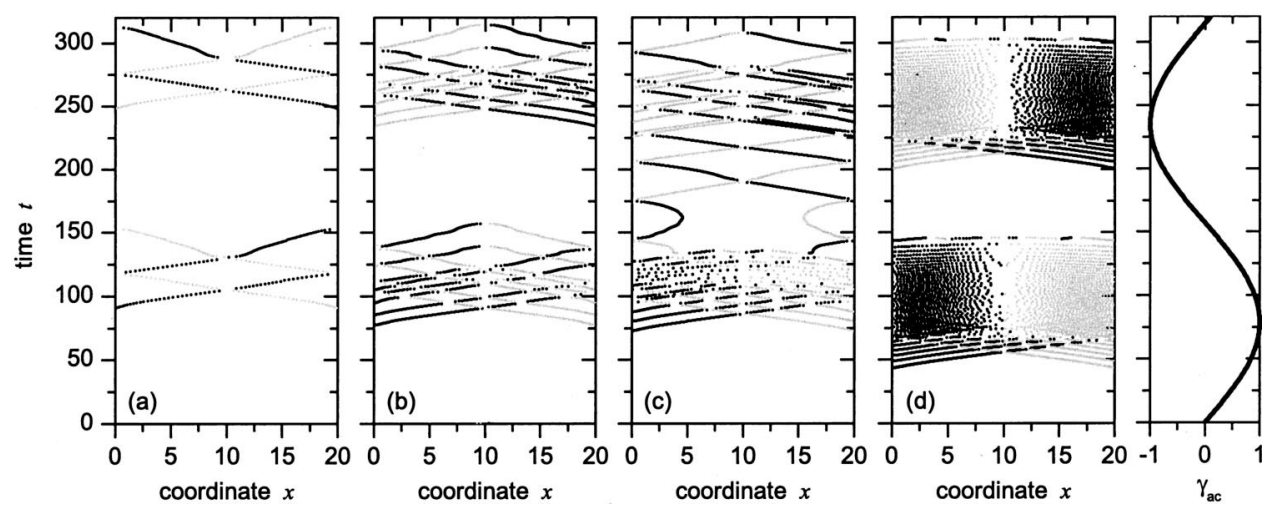

FIG. 15. The fluxons trajectories for $L=20$ and $\alpha=0.2$. 
times from the edges. Depending on the instant value of $\gamma_{\mathrm{ac}}$, they either pass through each other in the center of the junction or just return back to the edge under the action of the bias current. Later, this F-AF pair joins the dynamics of the fluxons injected during the negative semiperiod of ac drive, as a result of which all $\mathrm{F}-\mathrm{AF}$ pairs finally annihilate. The detailed investigation of the trajectories connecting positive and negative semiperiods of ac drive is beyond the scope of this article, but we predict that the difference between trajectories shown in Fig. 15(c) and Figs. 15(a), 15(b), and 15(d) results in a qualitatively different content of the second harmonic generated by our nonlinear system, since we have spontaneous breaking of the symmetry between positive and negative semiperiods.

Finally, as an example, in Fig. 15(d) we show the fluxon motion at rather high $\gamma_{\mathrm{ac}}=0.80$. There are two fluxes of solitons and antisolitons from the left and from the right sides which pass through each other and reach the opposite edge of the LJJ, where the injection of the new (anti-)fluxons still goes on. Upon reflection, which changes the polarity of the solitons, the flux of the solitons and antisolitons becomes twice as intense as before. When these two fluxes collide again, the massive annihilation takes place, as a result of which no fluxon survives. The same happens during the negative semiperiod.

\section{CONCLUSION}

We have studied numerically the fluxon dynamics and have calculated the complex impedance of a LJJ embedded into a resonator for the case of a finite capacitance of a LJJ, different LJJ length, damping, additional dc bias current, and external dc magnetic field. The right choice of discretization used for simulation is discussed. The finite capacitance can make the reactance of the junction negative and allows for some tuning of the resonator. We have found a crossover between moderate and very long junction length, which corresponds to the crossover between strong coupling and decoupling of two dissipation mechanisms present in the system. The crossover length is $\propto \alpha^{-1}$ for $\alpha \geqslant 1$, and $L$ $\approx 2 \pi / \omega \bar{c}_{0}$ for $\alpha \ll 1$. The presence of two decoupled dissipa- tion mechanisms may result in nonmonotonic dependence of $R\left(\gamma_{\mathrm{ac}}\right)$, as we have demonstrated for different cases. The dc bias current introduces the asymmetry into the fluxon dynamics picture which results in higher losses at the same amplitude of $\gamma_{\mathrm{ac}}$. The application of the magnetic field results in fluxon trapping and hysteretic behavior of the impedance as a function of the field. Although it is difficult to get rid of the trapped vortices manipulating with the field, the dependence of the resistance on the field $R(H)$ shows a set of the characteristic peaks which are associated with the individual (pairs of) vortices entering or leaving the LJJ. Thus the measurement of the microwave impedance at low pumping levels may be an interesting technique to detect (and even count the number of) trapped fluxons in the LJJ and related systems. In our opinion, new measurements of the impedance of HTS in the presence of the dc current or external magnetic field may give additional insights into the nature of losses in HTS.

\section{ACKNOWLEDGMENTS}

The authors thank A. Ustinov, D. Oates, and J. Halbritter for encouraging discussions and P. Caputo for careful proofreading of the manuscript and valuable suggestions.

${ }^{1}$ T. L. Hylton and M. R. Beasly, Phys. Rev. B 39, 9042 (1989).

${ }^{2}$ D. E. Oates, P. P. Nguyen, Y. Habib, G. Dresselhaus, M. S. Dresselhaus, G. Koren, and E. Polturak, Appl. Phys. Lett. 68, 705 (1996).

${ }^{3}$ C. J. Lehner, D. E. Oates, Y. M. Habib, G. Dresselhaus, and M. S. Dresselhaus, J. Supercond. 12, 363 (1999).

${ }^{4}$ Y. M. Habib, C. J. Lehner, D. E. Oates, L. R. Vale, R. H. Ono, G. Dresselhaus, and M. S. Dresselhaus, Phys. Rev. B 57, 13833 (1998).

${ }^{5}$ T. C. L. G. Sollner, J. P. Sage, and D. E. Oates, Appl. Phys. Lett. 68, 1003 (1996).

${ }^{6}$ H. Xin, D. E. Oates, S. Sridhar, G. Dresselhaus, and M. S. Dresselhaus, Phys. Rev. B 61, 14952 (2000).

${ }^{7}$ D. E. Oates, H. Xin, G. Dresselhaus, and M. S. Dresselhaus, IEEE Trans. Appl. Supercond. 11, 2804 (2001).

${ }^{8}$ E. Goldobin, available online: http://www.geocities.com/SiliconValley/ Heights/7318/StkJJ.htm (2000)

${ }^{9}$ D. M. Sheen, S. M. Ali, D. E. Oates, R. S. Withers, and J. A. Kong, IEEE Trans. Appl. Supercond. 1, 108 (1991).

${ }^{10}$ T. Van Duzer and C. W. Turner, Principles of Superconducting Devices and Circuits (Prentice Hall, Englewood Cliffs, NJ, 1998).

${ }^{11}$ V. I. Karpman, E. M. Maslov, and V. V. Solov'ev, Sov. Phys. JETP 57, 167 (1983) 